\title{
Combination of adaptive enlargement and reduction in the search neighbourhood in the bees algorithm
}

\begin{abstract}
The Bees Algorithm, a heuristic optimisation procedure that mimics bees foraging behaviour, is becoming more popular among swarm intelligence researchers. The algorithm involves neighbourhood and global search and is able to find promising solutions to complex multimodal optimisation problems. The purpose of neighbourhood search is to intensify the search effort around promising solutions, while global search is to enable avoidance of local optima. Despite numerous studies aimed at enhancing the Bees Algorithm, there have not been many attempts at studying neighbourhood search. In this work, the combination of adaptive enlargement and reduction of the search neighbourhood is presented. Two engineering design problems with constraints which were the pressure vessel and speed reducer were selected to demonstrate the performance of the modified algorithm. The experimental results obtained showed that this combination is beneficial to the proposed algorithm.
\end{abstract}

Keyword: Adaptive enlargement; Search neighbourhood; The bees algorithm 